\title{
QT Interval Dispersion Behavior in Patients with and without Obstructive Coronary Artery Disease Undergoing Exercise Test
}

\author{
Alexandre Maulaz Barcelos, ${ }^{1 \oplus}$ Marcelo Perim Baldo, ${ }^{2 \oplus}$ Sérgio Lamego Rodrigues, ${ }^{2 \oplus}$ José Geraldo Mill ${ }^{\circledR}$ \\ CEAP - Centro de Ensino e Aperfeiçoamento em Pesquisa - Hospital Evangélico de Vila Velha, 'V Vila Velha, ES - Brazil \\ Universidade Federal do Espírito Santo (UFES), ${ }^{2}$ Espírito Santo, ES - Brazil
}

\section{Abstract}

Background: Stress test is used to detect coronary artery disease (CAD). The QTc interval dispersion (dQTc) is an electrocardiographic index of ventricular repolarization heterogeneity. Some researchers have linked transient myocardial ischemia induced by physical exertion with increased heterogeneity of ventricular repolarization measured by dQTc.

Objectives: To study the patterns of dQT in patients with and without chronic obstructive CAD and to define a reliable cutoff point for dQT that could become a diagnostic criterion for myocardial ischemia.

Methods: We retrospectively analyzed the electrocardiogram in resting and in exercise of 63 patients submitted to exercise test and cardiac catheterization. We divided the patients into three groups: true negative (VN), true positive (VP) and false positive (FP). VN: patients with coronary lesion lower than $70 \%$ and exercise test without myocardial ischemia; VP: individuals with stenosis greater than $70 \%$ in coronary arteries and a test suggestive of myocardial ischemia; FP: people with stenosis lower than $70 \%$ in the coronary arteries and stress test with ischemia criteria. Values of $\mathrm{p}<0.05$ were considered statistically significant.

Results: Resting dQTc was not different among the three groups. However, for the dispersion of the QTc interval in exercise was, respectively, $47 \pm 17 \mathrm{~ms}, 72 \pm 42 \mathrm{~ms}$, and $61 \pm 31 \mathrm{~ms}$ for VN, VP and FP (p=0.003).

Conclusions: Obstructive chronic coronary disease patients have an increase in dQTc during exercise. Measurement of dQTc may be helpful in the diagnosis of myocardial ischemia in the stress test. (Int J Cardiovasc Sci. 2020; 33(3):263-271)

Keywords: Coronary Artery Disease/physiopathology; Exercise Test/method; Electrocardiography/method; Myocardial Ischemia; Electrophysiology; QT Dispersion Interval.

\section{Introduction}

Stress test has been used in Brazil since 1972 and its sensitivity and specificity for the diagnosis of chronic CAD are $50-72 \%$ and $69-74 \%$, respectively. ${ }^{1-4}$ The QT interval dispersion (QTD) measurement is considered a promising instrument to improve the diagnostic accuracy of stress test. QTD was defined in the $1990 \mathrm{~s}^{5}$ as the difference between maximal and minimal QT interval duration measured in 12 ECG leads. It has been proposed as a regional marker of ventricular repolarization dispersion (VRD) and correlates with the dispersion of action potentials (AP) in animals and humans. ${ }^{6}$ QT interval, measured from the beginning of the QRS complex to the end of the $\mathrm{T}$ wave, represents the time it takes for ventricular myocardial cells to depolarize and repolarize. ${ }^{7}$ However, U-wave should not be included in the measurement. ${ }^{8}$

During exertion, patients with chronic CAD present increased ventricular repolarization heterogeneity,

Mailing Address: Alexandre Maulaz Barcelos

R. Vênus, s/n - Industrial do Alecrim. Postal Code: 29118-300, Vila Velha, ES - Brazil.

E-mail: alexandremaulazbarcelos@gmail.com 
which is reflected by increased QTD. ${ }^{9-11}$ Koide et al., ${ }^{12}$ observed that even when a coronary patient, undergoing stress test, did not present ischemia criteria, the QTD was higher $(62 \pm 13 \mathrm{~ms})$ compared to patients without coronary disease (40 $\pm 14 \mathrm{~ms})$. Musha et al., ${ }^{13}$ and col. showed an increase of QTD after exercise, which was not reduced by beta-blockers. ${ }^{13}$ Naka,et al., ${ }^{14}$ in a study with infarcted patients, found an increase in QTD due to residual ischemia;however, it did not increase in patients without residual ischemia.

There are several limitations to QT interval measurement technique. Among them, T-P fusion during higher heart-rates and changes in Qt interval rates in relation to men and women ${ }^{15,16}$ are worthy of note.

The aims of this study are to evaluate whether the QTD index is sensitive to action potential changes in the presence of stress induced myocardial ischemia, as well as to define a cutoff point for QTD that could become a diagnostic criterion for myocardial ischemia.

\section{Methods}

An observational analytical study, where 80 patients underwent exercise testing and coronary angiography (CAT), with a maximum interval of 6 months between the tests. The patients were aged 18 to 80 years. People who had any of the following conditions were excluded from the study: previous acute myocardial infarction (AMI), complete right [RBBB] or left [LBBB] bundle branch block, patients with long QT syndrome; patients with known ventricular dysfunction; unreadable ECG traces or ECG where less than eight electrocradiographic leads were available for QTI measurement. ${ }^{17}$ A total sample of 80 patients was defined at the convenience of the researcher.

Treadmill test was performed with an analog-to digital converter of signals, Ergo PC 13 model in Micromed 2.3 version, with a simultaneous acquisition of twelve leads and record with speed of $25 \mathrm{~mm} / \mathrm{s}$ and amplitude of $10 \mathrm{~mm} / \mathrm{mV}$. The protocols used for all patients were individualized aiming at reaching maximum heart rate. The test was considered suggestive of myocardial ischemia in case the patient presented at least onde one the ischemia criteria defined by the III Guidelines on ergometric tests of the Brazilian Society of Cardiology; ${ }^{18}$ typical chest pain on exertion;ST-segment elevation or depression, equal to or greater than $1 \mathrm{~mm}$, in relation to baseline ECG. The QT interval of each lead was calculated by the mean of the three beats with less artifact. By using a cursor, one point was marked at the beginning of the
QRS complex and another at the end of the T-wave (the point where the $\mathrm{T}$ wave returned to the isoelectric line) for each of the three beats. ${ }^{11}$ After the measurement, we calculated the mean of the three values found, which would be the value to be considered as the QTI of the mentioned lead. The same procedure was performed for the 12 leads. Thus, for each patient in the study, at least, 24 QT intervals were measured at rest (standing) and 24 on ECG obtained within the first minute of the recovery stage. We decided to "measure the peak stress" within the first minute of the recovery phase in order to minimize the technique's artifacts. At the end of the measurement of the QT intervals of all leads, we marked the highest and the lowest measure found, in the two phases studied: rest and effort. From these values, we calculated the QTI dispersion of these two phases, and also a delta QT dispersion value by determining the difference of QTD between effort and rest.

In order to adjust the QT interval for the corresponding heart rate (HR), we used the Bazett's formula. The adjustment enabled the calculation of the QTc (QT interval dispersion corrected for heart rate), and also the QTc "delta" - the difference between rest-stress QTc intervals. All the electrocardiographic measurements were done by a single observer. Figure 1 shows the sequence to measure a QT interval.

Interobserver variability was determined by measurements performed by a second researcher, who was blinded to the measures obtained by the first observer. The second ECG expert measured the QT interval in 12 patients randomly selected (patients were numbered from 1 to 63 , and 12 numbers were raffled). The correlation between the measures was determined by Pearson's correlation coeficiente. The Bland-Altman test was also used to assess interobserver variability (Figure 2).

After catheterization, patients with a coronary stenosis of at least $70 \%$ of one or more arteries, or with $\geq 50 \%$ stenosis of the left coronary trunk (LCT), were classified as "people with obstructive coronaropathy". In contrast, patients with with stenosis less than $70 \%$ in epicardial coronary arteries, or less than $50 \%$ in the left coronary trunk, were classified as "without obstructive coronary disease".

The Medcalc software was used for the statistical analysis. The data were expressed in absolute numbers, percentages and standard deviation. The classificatory variables were presented as tables, and the propotions were compared using the chi-square 

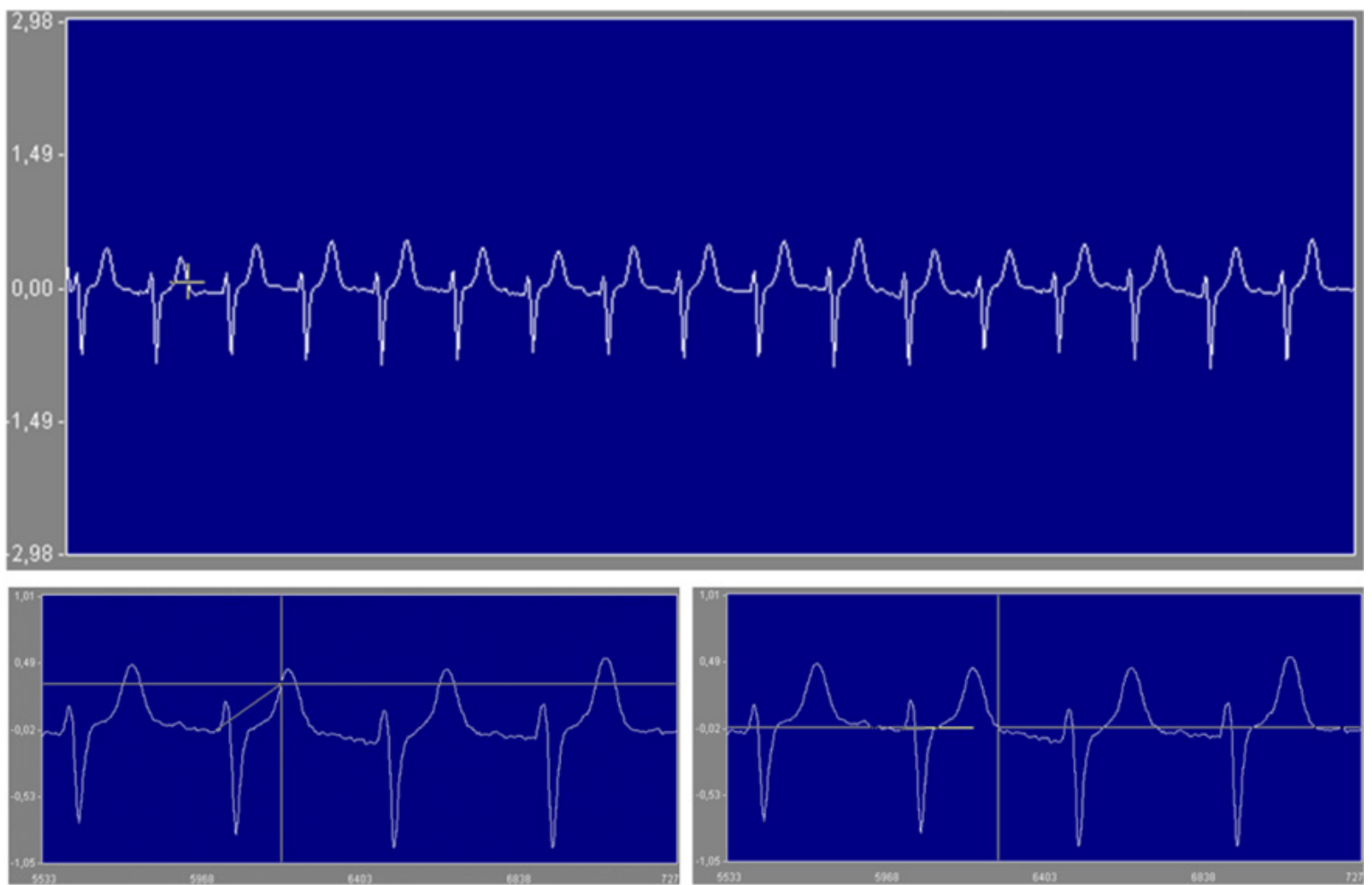

Figure 1 - Measurement sequencing of a QT interval.

A

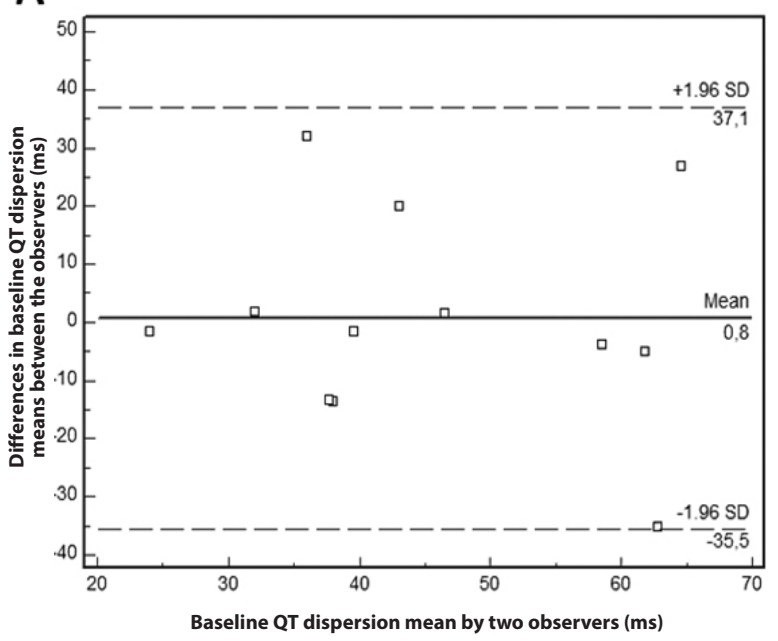

B

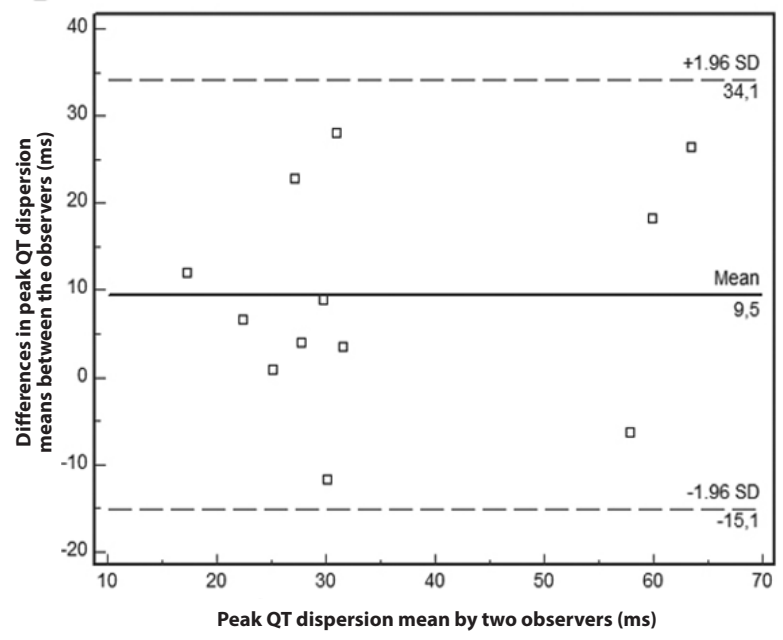

Figure 2 - Reproducibility of QT dispersion measurements before (A) and during (B) effort, assessed using the Bland-Altman method. 
test. The Kolmogorov-Smirnov was used to assess the normality of the continuous variables. To identify the best QT dispersion cutoff point for the diagnosis of obstructive coronaropathy, ROC curves were used both for QT dispersion values and for rest and stress QTc. We calculated the QTd, the QTc, and conventional stress test sensitivity and specificity for the diagnosis of chronic obstructive coronaropathy. We also found a QTc delta - the difference between rest-stress QTc intervals, as well as the QT delta - the difference between rest-stress QTd intervals. Subsequently, patients were divided into three groups: true positive $(\mathrm{TP})$ - patients with positive stress test for ischemia and coronary angiography showing stenosis $\geq 70 \%$ of at least one major epicardial artery, except for left coronary trunk lesions which were considered to be significant when the obstruction was $>50 \%$. The false positive (FP) group was composed of patients with positive stress test and stenosis less than $70 \%$ in at least one major epicardial artery, except for left coronary trunk lesions which were considered to be significant when the obstruction was $<50 \%$. Finally, the true negative (TN) group was composed of negative stress test patients and coronary angiography showing stenosis less than 70\% in any epicardial coronary artery, except for left coronary trunk lesions which were considered to be significant when the obstruction was $<50 \%$. p $<0.05$ was considered to be statistically significant and one-way-variance analysis (ANOVA) was used to compare the three groups. A paired t-test was used to assess the QTc behavior during rest and effort.

All the patients signed the free and clarified term of consent and the research Project was approved by the Ethics Committee on Human Research of Federal University of Espirito Santo (UFES), by the protocol number: 06177412.1.0000.5071.

\section{Results}

The difference between the mean QTd values obtained by both observers, at rest, was only $0,8 \pm 18,3$ ms, which is quite satisfactory. However, we observed some data points of standard deviation away from the mean, which demonstrated the low reproducibility of the measures at rest. The difference between the means obtained by the two observers was $9,5 \pm 12,5 \mathrm{~ms}$ in peak stress measurements. Additionally, we also found data points of standard deviation away from the mean, which confirmed the low reproducibility of QT dispersion for peak stress measures as well.
The variability between the two observers was also analyzed using Pearson's correlation coefficient. For the baseline value of QTd, the value of " $\mathrm{r}$ " found was 0.36 $(p=0.25)$. For the peak QTd, the value of " $r$ " was 0.73 $(p=0.007)$. The correlation between the measures obtained by the two observers was positive weak at rest and positive moderate on exertion. These results indicate a poor reproducibility of QT interval dispersion measures.

Out of the 74 patients initially selected, after we excluded those who presented any of the exclusion criteria, and after the false-positives were removed because they were of no interest for the research (negative stress test and stenosis greater than $70 \%$ in epicardial arteries, except for trunk lesions which were considered to be significant when the obstruction was $>50 \%$ ), there were 63 patients who fulfilled the criteria to be in the three research groups: TP, FP and TN. The three groups studied (TP, FP and TN) were similar in relation to general characteristics (Table 1), including comorbidities and medicaments in use.

The cutoff value found with the ROC curve was the point where better QTd and QTc sensitivity and specificity for the diagnosis significant coronaropathy were achieved (46ms and 57ms, respectively). Regarding

Table 1 - General characteristics of the groups

\begin{tabular}{|c|c|c|c|c|}
\hline & $\begin{array}{c}\text { TP } \\
(n=26)\end{array}$ & $\begin{array}{c}\text { FP } \\
(n=23)\end{array}$ & $\begin{array}{c}\text { TN } \\
(n=14)\end{array}$ & p-value \\
\hline Age (years) & $58 \pm 10$ & $54 \pm 12$ & $56 \pm 11$ & 0.43 \\
\hline Male sex (\%) & $81 \%$ & $71 \%$ & $71 \%$ & 0.453 \\
\hline $\begin{array}{l}\text { Diabetes } \\
\text { mellitus (\%) }\end{array}$ & $27 \%$ & $13 \%$ & $7 \%$ & 0.213 \\
\hline $\begin{array}{l}\text { Arterial } \\
\text { hypertension } \\
(\%)\end{array}$ & $58 \%$ & $58 \%$ & $79 \%$ & 0.372 \\
\hline $\begin{array}{l}\text { Beta-blockers } \\
(\%)\end{array}$ & $23 \%$ & $33 \%$ & $21 \%$ & 0.633 \\
\hline ACEI/ARB (\%) & $42 \%$ & $21 \%$ & $21 \%$ & 0.188 \\
\hline Statins (\%) & $12 \%$ & $8 \%$ & $21 \%$ & 0.199 \\
\hline $\begin{array}{l}\text { Calcium } \\
\text { antagonists (\%) }\end{array}$ & $12 \%$ & $0 \%$ & $29 \%$ & \\
\hline $\begin{array}{l}\text { Values expressed } \\
\text { FP: false positive }\end{array}$ & $\begin{array}{l}\text { mean } \pm S I \\
\mathrm{~V}: \text { true ne }\end{array}$ & percentas & гP: true $p$ & \\
\hline
\end{tabular}


the stress QTd, the sensitivity was $44.4 \%$ and the specificity was $81.6 \%$ (AUC 0.585;CI 95\% 0.465-0.699) with a cutoff of $46 \mathrm{~ms}$. For stress QTc, the sensitivity was $58.3 \%$ and the specificity was $63.2 \%$ (AUC $0.593 ; \mathrm{CI}$ $95 \% 0.472-0.706)$ with a cutoff of $57 \mathrm{~ms}$. In relation to the sensitivity and specificity values of the traditional treadmill test, and considering the presence of ST segment depression or typical chest pain on exertion, we found a sensitivity of $72 \%$ and a specificity of $32 \%$ in our sample. Since the confidence intervals found for the QTd and QTc cutoff values included the 0.5 value, we decided not to aggregate the QT dispersion values into the traditional stress test, because any improvements in the sensitivity and specificity that we could possibly find would not have been reliable.

When we analyzed the three groups formed (TN, TP and FP), the following was found: the mean values of QT dispersion at rest did not show statistically significant difference between the three groups. Respectively, $58 \pm 30 \mathrm{~ms}, 47 \pm 22 \mathrm{~ms}$ and $43 \pm 19 \mathrm{~ms}$, for the TN, TP and FP groups $(p=0.172)$. In addition, we did not observe significant difference between the mean values of the QTc dispersion at rest: $67 \pm 40 \mathrm{~ms}, 55 \pm 26 \mathrm{~ms}$ and $49 \pm 21 \mathrm{~ms}$, respectively, for the TN, TP and FP groups $(p=0,163)$. Thus, we moved on to analyze the mean QT dispersion values found between the three groups during effort. Similarly to what happened in relation to QT dispersion at rest, we found close mean values with no statistical difference between the three groups: $32 \pm 11 \mathrm{~ms}$, $48 \pm 28 \mathrm{~ms}$ and $42 \pm 22 \mathrm{~ms}$, respectively, for the TN, TP and FP groups $(p=0.124)$. However, when we analyzed the data of QTc dispersion of effort, we verified that the values of QTc between the three groups were different: $\mathrm{TN}(47 \pm 17 \mathrm{~ms}), \mathrm{TP}(72 \pm 42 \mathrm{~ms})$ and FP $(61 \pm 31 \mathrm{~ms})$, with $\mathrm{p}=0.003$. When we compared TN and TP, we found $\mathrm{p}<0.05$; when comparing TN and FP, we also found $\mathrm{p}<0.05$; however, when VP and FP were compared, we found $\mathrm{p}>0.05$ (Table 2).

In order to better assess the changes in stress induced coronary depolarization, we created a delta QT dispersion value $(\triangle \mathrm{QTD})$ which was obtained by the following equation: $\Delta \mathrm{dQT}=\mathrm{QTd}$ stress - QTd rest. Likewise, we obtained a delta value of QTc dipersion by a similar equation: $\Delta \mathrm{dQTc}=\mathrm{QTc}$ stress $-\mathrm{QTc}$ rest. The $\triangle \mathrm{dQT}$ was $-25 \pm 33 \mathrm{~ms}$ in the TN group, $1 \pm 27 \mathrm{~ms}$ in the TP and $-2 \pm 23 \mathrm{~ms}$ in the FP group, with statistical difference between the three groups, with $p=0.013$. Comparing TN and TP, we found $\mathrm{p}<0.05$; the same was observed when TN was compared with FP ( $p<0.05)$;in

\begin{tabular}{|c|c|c|c|c|}
\hline & TP & FP & $\mathrm{TN}$ & p-value \\
\hline $\begin{array}{l}\text { Number of } \\
\text { patients }\end{array}$ & 26 & 23 & 14 & \\
\hline Rest HR (bpm) & $77 \pm 13$ & $79 \pm 11$ & $79 \pm 14$ & $\mathrm{p}=0.922$ \\
\hline Peak HR (bpm) & $134 \pm 15$ & $131 \pm 21$ & $128 \pm 22$ & $p=0.637$ \\
\hline Peak SP (mmHg) & $171 \pm 21$ & $173 \pm 39$ & $193 \pm 29$ & $\mathrm{p}=0.071$ \\
\hline $\begin{array}{l}\text { Chest pain on } \\
\text { exertion (\%) }\end{array}$ & $53 \%$ & $45 \%$ & 0 & \\
\hline $\begin{array}{l}\text { ST depression } \\
(\%)\end{array}$ & $88,40 \%$ & $79,20 \%$ & 0 & \\
\hline $\begin{array}{l}\text { Pain with ST } \\
\text { depression (\%) }\end{array}$ & $42,3 \%$ & $25 \%$ & 0 & \\
\hline Rest QTd (ms) & $47 \pm 22$ & $43 \pm 19$ & $58 \pm 30$ & $p=0.172$ \\
\hline Rest QTc (ms) & $55 \pm 26$ & $49 \pm 21$ & $67 \pm 40$ & $p=0.163$ \\
\hline Peak QTd (ms) & $48 \pm 28$ & $42 \pm 22$ & $32 \pm 11$ & $p=0.124$ \\
\hline Peak QTd (ms) & $72 \pm 42$ & $61 \pm 31$ & $47 \pm 17$ & $p=0.003$ \\
\hline$\Delta \mathrm{QTD}(\mathrm{ms})$ & $1 \pm 27$ & $-2 \pm 23$ & $-25 \pm 33$ & $\mathrm{p}=0.013$ \\
\hline$\Delta \mathrm{QTDc}(\mathrm{ms})$ & $17 \pm 40$ & $11 \pm 30$ & $-20 \pm 45$ & $p=0.013$ \\
\hline \multicolumn{5}{|c|}{$\begin{array}{l}Q T d: Q T \text { dispersion; } Q T c: Q T \text { dispersion corrected for heart rate; } \\
\triangle Q T D: \text { Stress peak } Q T \text { dispersion minus rest } Q T \text { dispersion; } \triangle Q T D c: \\
Q T \text { dispersion corrected for stress peak HF minus QT dispersion } \\
\text { corrected for HF at rest. }\end{array}$} \\
\hline
\end{tabular}

contrast, the comparison between TP and FP showed $\mathrm{p}>0.05$. The mean $\triangle \mathrm{QTC}$ dispersion was $-20 \pm 45$ $\mathrm{ms}$ in the TN group, $17 \pm 40 \mathrm{~ms}$ in the TP group and $11 \pm 30 \mathrm{~ms}$ in the FP group. Again, the same " $\mathrm{p}$ " value of 0.013 was found between the three groups, as well as the same values of " $\mathrm{p}$ " for the other comparisons: TN vs $\mathrm{VP}(\mathrm{p}<0.05), \operatorname{VN}$ vs FP $(\mathrm{p}<0.05)$ and VP vs FP $(\mathrm{p}>0.05)$. We did not find any statistical difference between TP vs FP. Due to the statistical difference found between the three groups, in relation to the mean values of $\Delta \mathrm{QTc}$, we decided to illustrate the behavior of QTc dispersion from rest to stress peak in the three groups. Figures 3, 4 and 5 show the behavior of the three groups.

\section{Discussion}

The aim of this study was to assess the relationship between QT interval dispersion and chronic CAD. The focus of our investigation was to evaluate the feasibility 


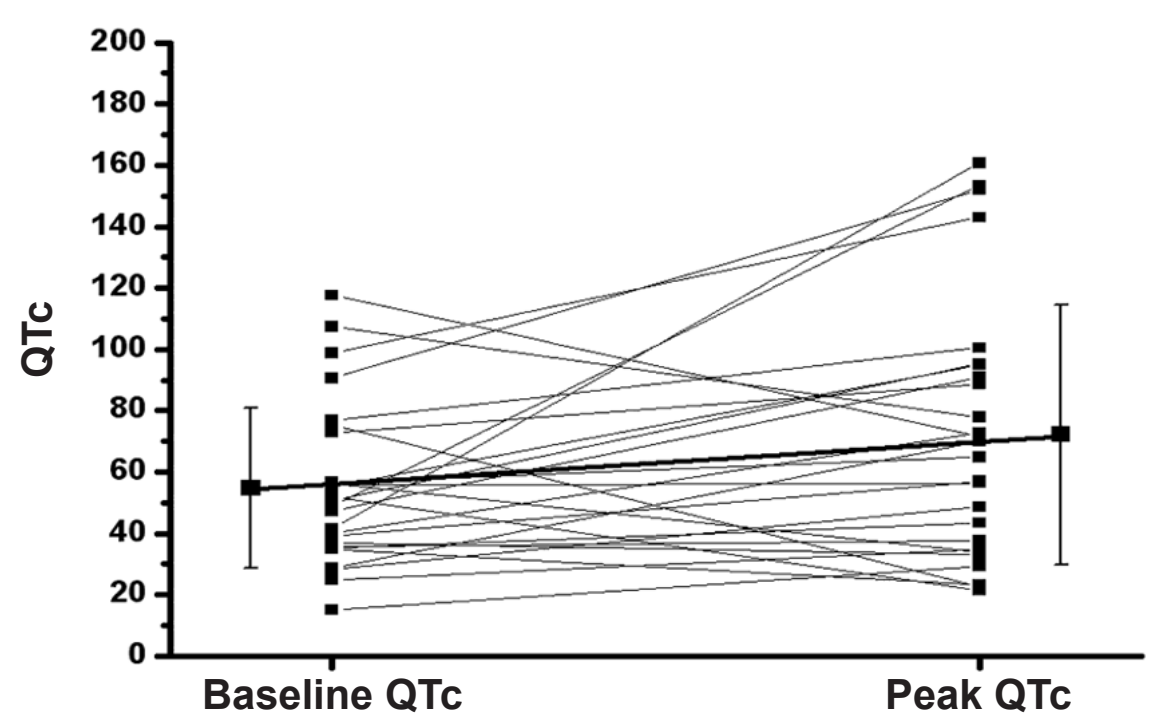

Figure 3 - QTc evolution in the true positive group from rest (baseline QTc dispersion) to effort (peak QTc dispersion) conditions.

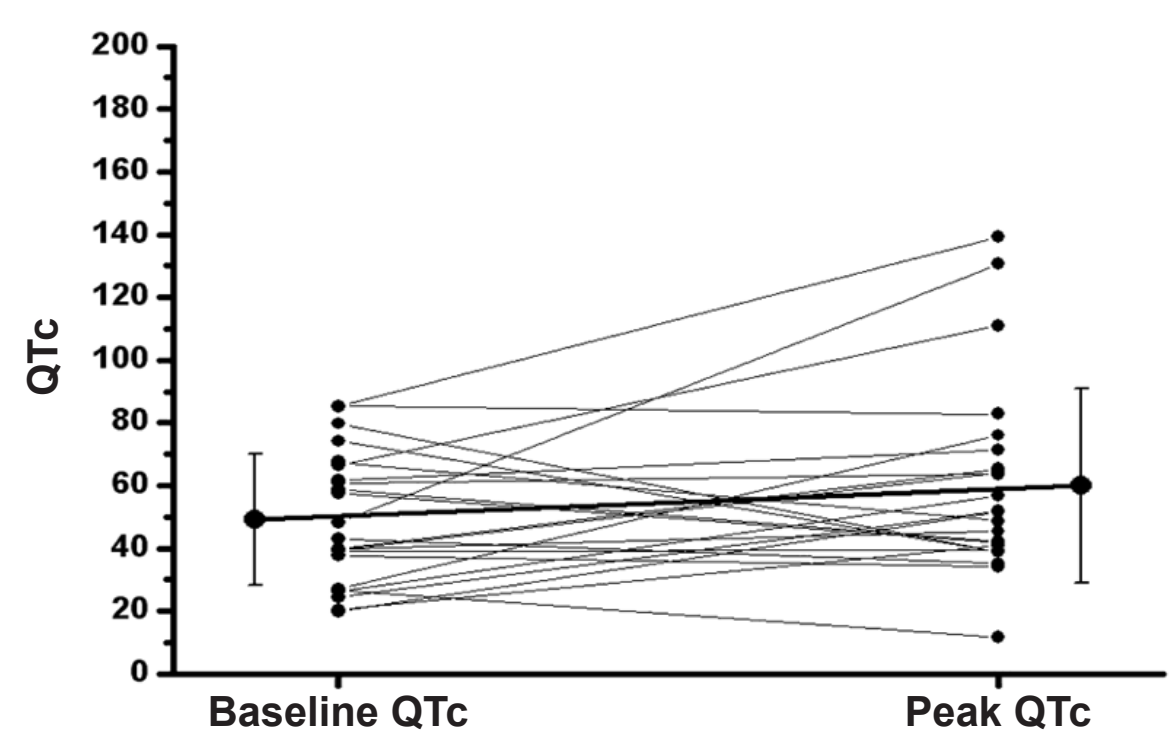

Figure 4 - QTc evolution from rest (baseline QTc) to effort (peak QTc) conditions in the false positive group.

of using the QTd (within the first minute of recovery and/or rest) for the diagnosis of significant coronary disease. Based on previous studies, we hypothesized that coronary obstruction would lead to prolonged action potential in the ischemic region, and that such increase could be identified by the QTd. Actually, it would reflect in theory the difference between repolarization in the ischemic region compared to the non-ischemic in the ventricular syncytium.

The first result that will be discussed concers the reproducibility of QT dispersion measurements. One of the major problems concerning QT interval measurements is the difficulty in obtaining acceptable interobserver variability. In our study, interobserver 


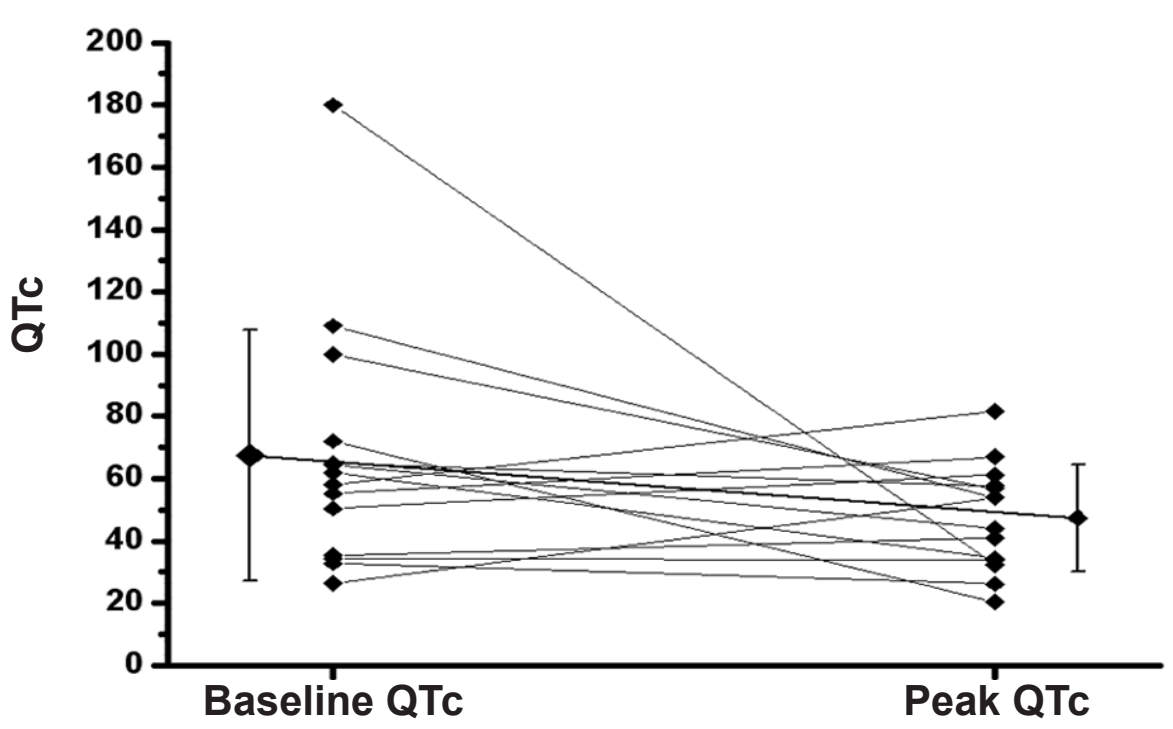

Figure 5 - QTc evolution in the true negative group from rest (baseline QTc) to effort (peak QTc) conditions.

reproducibility was weak. Our impression is that, even with the measurements being performed with more modern software, the problem involving the reproducibility of QT intervals measurements remains a considerable one.

With the results of catheterization, QT dispersion, QTc and stress test, we built ROC curves in order to find the possible cutoff values of QTd and and stressrest QTc, so as to subsequently calculate the sensitivity and specificity of QTd for the diagnosis of chronic CAD. We did not achieve a minimaly satisfactory ROC curve for QTd and QTc at rest. For QT and QTc dispersion of effort, we obtained ROC curves somewhat better than those obtained at rest, and we found cutoff values for QTd (46 ms) and QTc (57 ms). As for the sensitivity and specificity of stress QTd and QTc it is possible to say that they were comparable to the traditional stress test ischemic criteria. However, what really called attention, was the low specificity of the classical diagnostic criteria of exercise induced ischemia in our sample (32\%). On the other hand, the sensitivity was $72 \%$. The high falsepositive rate was quite high. We observed that most of the false-positive results were found in the presence of segment depression without a concomitant stenosis of at least $70 \%$. The most plausible explanation found lies in the arbitrariness of considering as the possible cause of ischemia only stenosis with obstruction greater than $70 \%$. Smaller plaques, but under the effect of vasoconstriction substances, can cause ischemia. Another factor to be considered is the possibility of microcirculation disease in patients without significant disease of large coronary arteries. Finally, we must consider that the degree quantification of coronary obstruction made by the doctor responsible for the catheterization is performed by visual method only and, hence, it is observer-dependent.

When we analyzed the data from the three groups formed: true positive (TP), false positive (FP) and true negative (TN), heart rate (HR) and systolic pressure (SP) at stress peak were not statistically different between the three groups (Tabble 2). The incidente of typical chest pain and ST depression at peak stress were higher among the false-positive and true-positive groups, and was not present in the TN group, as we already expected. In relation to the QT dispersion and QTc dispersion at rest, there was no significant statistical difference between the three groups. In contrast, stress QTc was significantly higher among the TP and FP groups, compared with the TN group. The TP and FP groups behaved so similarly that made us wonder about the real importance of considering "people with significant coronary artery disease" only those patients with stenosis of at least 70\% in epicardial arteries, or $50 \%$ or more in the left coronary trunk. We can speculate that if myocardial perfusion scintigraphy had been performed, instead of cardiac catheterization, as gold standard for significant CAD, it is possible that our results would have been similar to those 
obtained by Stoletniy and col.8. These authors showed that the QTc increased from rest to stress in patients with ischemia documented by myocardial scintigraphy, and that it did not increase from rest to stress peak in patients without myocardial scintigraphy documented using radioisotope techniques. We speculate that QTC is, ultimately, a marker of myocardial ischemia, with no strict connection with the degree of obstruction in large coronary arteries.

The QTc delta also showed significant statistical difference between the groups. However, in order to test the theory that supports the concept of QTd, we decided to scketch a line graph that represented each individual in the three groups. Our concern was that the QTc and QTc delta values would show only the statistical difference between the means of the three groups (extreme QTc values of few individuals of one group can affect the mean and not necessarily represent the behavior of the rest to stress condition variable). Corroborating the results of the QTc dispersion of effort, nineteen patients from the TP group presented increased QTc from rest to stress conditions (Figure 3), whereas seven individuals showed a reduction. In contrast, in the TN group, five people presented increased QTd and nine reductions (Figure 5). The FP group maintained similar behavior as the TP group. In FP, fourteen patients increased the QT dispersion and nine reduced it (Figure 4). In order to statistically test the behavior of each group in relation to the QTC dispersion of effort and at rest, we used a paired T-test for each group, and obtained significant difference between the TP group means from rest to stress conditions, and a a trend to increased QTc in the FP group. The TN negative group did not show significant changes between the QTc pre- and postexercise means. We conclude that QTc behaves, predominantly, with an increase on exertion in patients with myocardial ischemia;and tends not to change significantly in patients without ischemia.

Our study has some limitations that should be pointed out. First, our sample was small so we could not obtain a highly reliable cutoff point for QTc. Still, we must remember that our QTc cutoff point was quite similar to the ones found by other authors. Secondly, the methodology we used to measure the QTI - as far as we know - is unprecedented in the literature. Thus, our data must be confirmed by other similar studies. Our results can only be considered for a coronary population with no previous AMI or ventricular dysfunction. Finally, this is a database retrospective study with all the limitations inherent to this type of study.

\section{Conclusions}

Based on our results, we believe that QT dispersion - is spite of being a "crude" marker of ventricular repolarization heterogeneity - is sensitive to stressinduced myocardial ischemia and can aid in the diagnosis of chronic CAD.

\section{Author contributions}

Conception and design of the research: Barcelos AM, Mill JG. Acquisition of data: Barcelos AM, Rodrigues SL, Mill JG. Analysis and interpretation of the data: Barcelos AM, Baldo MP,Rodrigues SL, Mill JG. Statistical analysis: Barcelos AM, Baldo MP, Rodrigues SL, Mill JG. Obtaining financing: Barcelos AM. Writing of the manuscript: Barcelos AM. Critical revision of the manuscript for intellectual content: Barcelos AM, Baldo MP, Rodrigues SL, Mill JG.

\section{Potential Conflict of Interest}

No potential conflict of interest relevant to this article was reported.

\section{Sources of Funding}

There were no external funding sources for this study.

\section{Study Association}

This article is part of the thesis of master submitted by Alexandre Maulaz Barcelos, from CEAP - Centro de Ensino e Aperfeiçoamento em Pesquisa - Hospital Evangélico de Vila Velha.

\section{Ethics approval and consent to participate}

This study was approved by the Ethics Committee of the Hospital Universitário Cassiano Antônio de Moraes under the protocol number CAAE: 06177412.1.00007051. All the procedures in this study were in accordance with the 1975 Helsinki Declaration, updated in 2013. Informed consent was obtained from all participants included in the study. 


\section{References}

1. Detrano R, Gianrossi R, Froelicher V. The diagnostic accuracy of the exercise electrocardiogram: a meta-analysis of 22 years of research. Prog Cardiovasc Dis. 1989;32(3):173-206.

2. Gianrossi R, DetranoR, Mulvihill D, Lehmann K, DubachP, Colombo A, et al. Exercise-induced ST depression in the diagnosis of coronary artery disease: a meta-analysis. Circulation. 1989;80(1):87-98.

3. LadenheimML, KotlerTS, PollockBH, BermanDS, DiamondGA. Incremental prognostic power of clinical history, exercise electrocardiography, and myocardial perfusion scintigraphy in suspected coronary artery disease. Am J Cardiol. 1987;59(4):270-7.

4. Ellestad MH, Wan MK. Predictive implications of stress testing. Followup of 2700 subjects after maximum treadmill stress testing. Circulation. 1975;51(2): 363-9.

5. Day CP, McComb JM, Campbell RW. QT dispersion: an indication of arrhythmia risk in patients with long QT intervals. Br Heart J. 1990;63(6):342-4.

6. Zabel M, Portnoy S, Franz MR. Electrocardiographic indexes of dispersion of ventricular repolarization: anisolated heart validation study. J Am Coll Cardiol. 1995;25(3):746-52

7. Kautzner J. QT interval measurements. Card Electrophysiol Rev. 2002;6(3):273-7

8. Bonow RO, Mann DL, Zipes DP, Libby P. Braunwald's heart disease: a textbook of cardiovascular medicine. 7th. Philadelphia: W.B. Saunders; 2005. p. 2183

9. Stierle U, Giannitsis E, Sheikhzadeh A, Krüger D, Schmücker G, Mitusch $\mathrm{R}$, et al. Relation between QT dispersion and the extent of myocardial ischemia in patients with three-vessel coronary artery disease. Am J Cardiol. 1998;81(5):564-8.
10. Stoletniy LN, Pai RG. Valueof QT dispersion in the interpretation of exercise stress test in women. Circulation. 1997;96(3):904-10.

11. Sporton SC, Taggart P, Sutton PM, Walker JM, Hardman SM. Acute ischemia: a dynamic influence on QT dispersion. Lancet. 1997;349(9048):306-9.

12. Koide Y, Yotsukura M, Yoshino H, Ishikawa K. Usefulness of QT dispersion immediately after exercise as an indicator of coronary stenosis independent of gender or exercise-induced ST-segment depression. Am J Cardiol. 2000;86(12):1312-7.

13. Musha H, Kunishima T, Awaya T, Iwasaki T, Nagashima J, Nakamura $\mathrm{T}$, et al. Influence of exercise on QT dispersion in ischemic heart disease. Jpn Heart J. 1997;38(2):219-26.

14. Naka M, Shiotani I, Koretsune Y, Imai K, Akamatsu Y, Hishida E, et al. Occurrence of sustained increase in QT dispersion following exercise in patients with residual myocardial ischemia after healing of anterior wall myocardial infarction. Am J Cardiol. 1997;80(12):1528-31.

15. Chauhan VS, Krahn AD, Walker BD, Klein GJ, Skanes AC, Yee R. Sex differences in QTc interval and QT dispersion: dynamics during exercise and recovery in healthy subjects. Am Heart J. 2002;144(5):858-64.

16. Kligfield P, Lax KG, Okin PM. QT interval-heart rate relation during exercise in normal men and women: definition by linear regression analysis. J Am Coll Cardiol. 1996;28(6):1547-55.

17. Surawicz B, Knilans TK. Chou's electrocardiography in clinical pratice: adult \& pediatric. 5nd. Philadelphia: W.B. Saunders; 2001. p. 709.

18. Meneghelo RS, Araújo CG, Stein R, Mastrocolla LE, Albuquerque PF, Serra SM, et al. Sociedade Brasileira de Cardiologia. III Diretrizes da Sociedade Brasileira de Cardiologia sobre Teste Ergométrico. Arq Bras Cardiol. 2010;95(5 Suppl 1):1-26. 